\title{
VI.
}

\section{Die Fascia umbilicalis und deren Beziehung zum Nabelringbruch bei Kindern.}

\author{
Von Dr. med. H. Sachs in Dorpat.
}

(Hierzu Taf. IV-V.)

Unter den verschiedenen Brucharten nehmen die Nabelbrüche des Kindesalters - besser Nabelringbrüche, denn ich spreche hier nur von den erworbenen Nabelbrüchen - eine ganz besondere Stellung ein, gleichviel, ob wir sie vom theoretischen oder vom praktischen Standpunkte aus betrachten. Während für die Entstehung der Leisten- und Schenkelbrüche die Bedeutung des intraabdominalen Druckes von verschiedenen Seiten noch angezweifelt wird, behaupten die Nabelbrüche hierin eine Ausnahmestellung, insofern als bei ihnen die Möglichkeit einer Entstehung durch Ausstülpung des Bauchfells von innen her (nicht durch Zug von aussen) selbst von absoluten Gegnern der Drucktheorie zugestanden wird. Andererseits bieten die Nabelbrüche der Kinder durch die Leichtigkeit, mit welcher sie zur definitiven Heilung gebracht werden, sowie dureh die Seltenheit der Einklemmung auch in praktischer Beziehung gewisse Eigenthümlichkeiten.

Abgesehen davon, dass die grosse Mehrzahl der Nabelbrüche der ärztlichen Beobachtung ganz entgeht, so dass wir bis jetzt noch keine sichere Statistik derselben besitzen, wird der Arzt in den meisten Fällen, wo er eine Nabelhernie zu Gesicht bekommt, sich mit dem Anlegen eines zweckentsprechenden Bruchbandes begnügen. Nur selten dürfte beim Kinde ein Eingriff nöthig werden, welcher dem Arzt einen, wenn auch unvollständigen Einblick in die anatomischen Verhältnisse gewäbrt. Wenn wir uns schliesslich vergegenwärtigen, wie selten Nabelbrüche bei Kindern zum Gegenstande einer speciellen pathologischanatomischen Untersuchung werden, - weil eine Nabelhernie bei 
Fig.2.

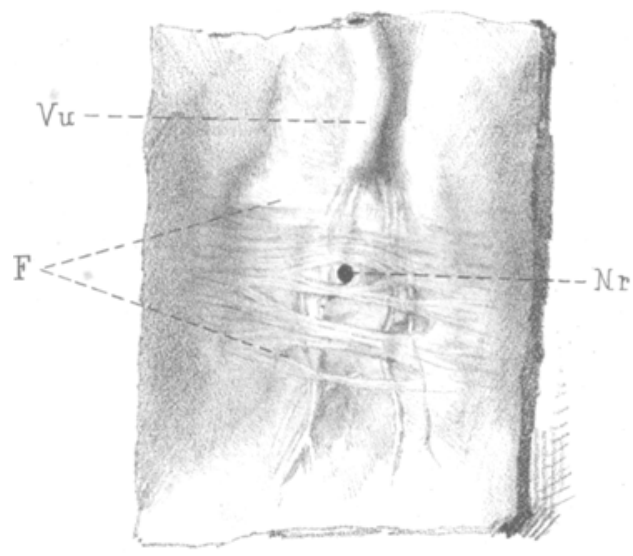

Fig 3.

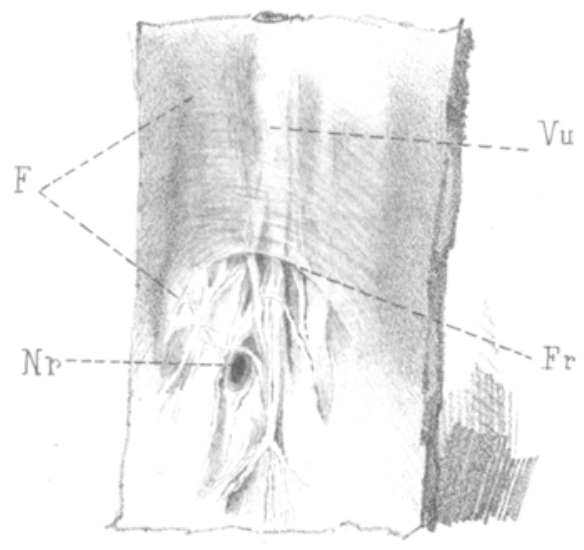

Piọ.1.

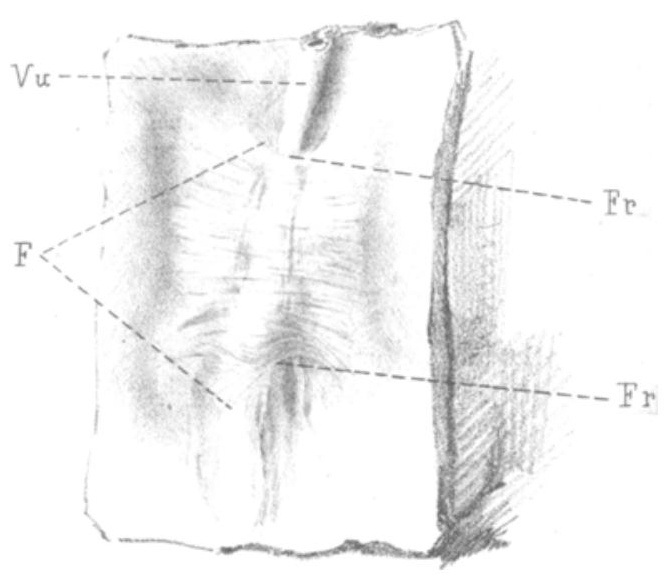

Figs.4.

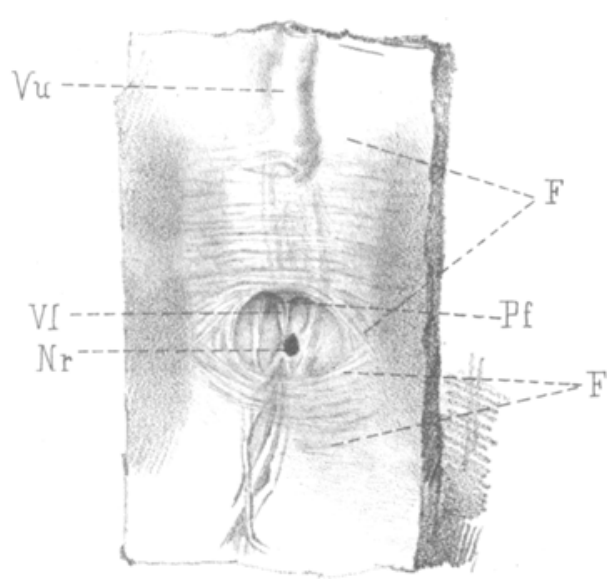


Fing.1.

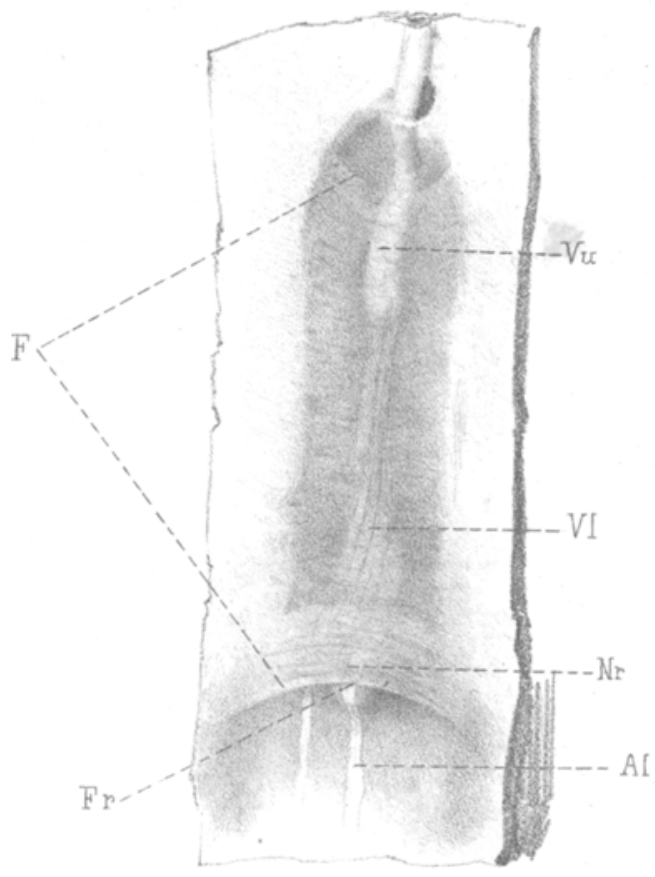

Fig. 3.

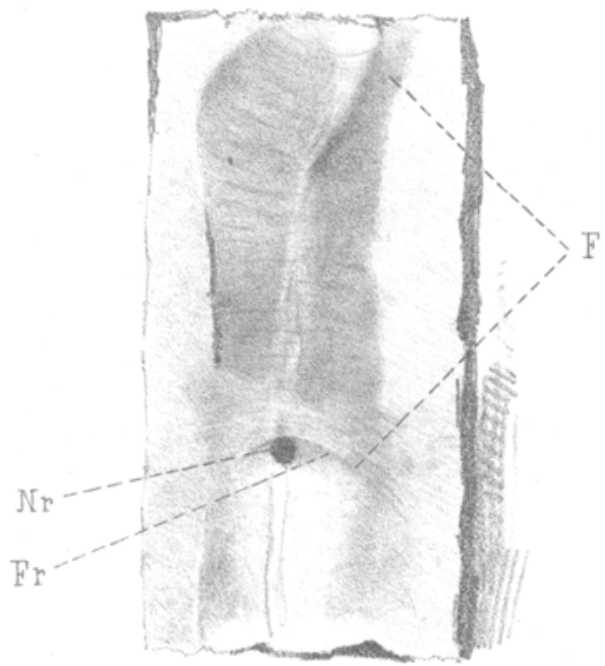

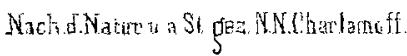

Fig. 2 .

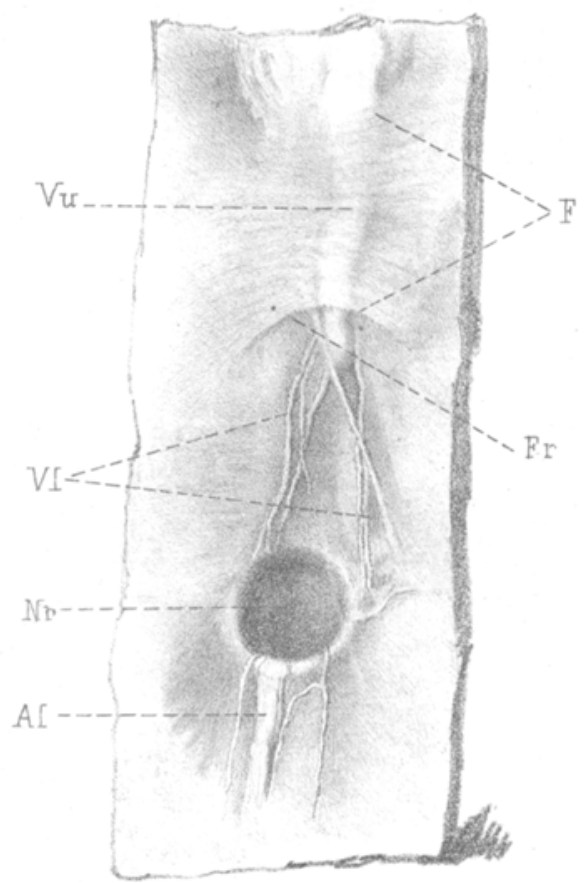

Fig. 4.

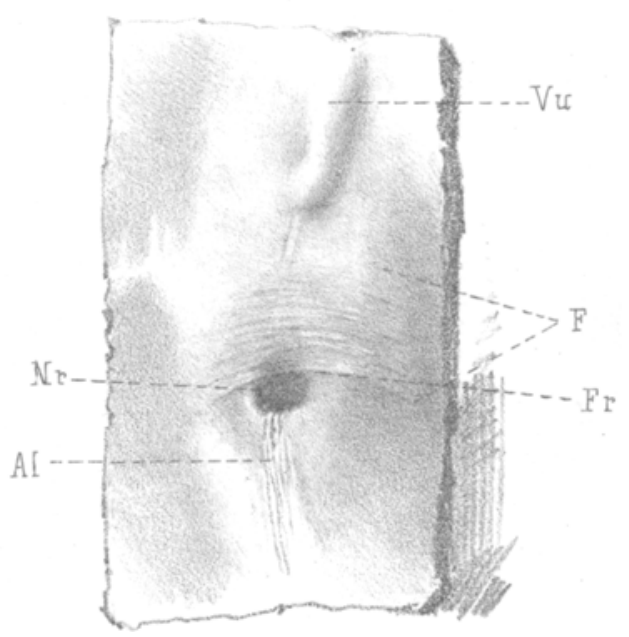

Lith Ch.Ivaneon SETetersber.

Petersb. Seite, gr. PB. Nol, 
Kindern nur selten den Tod veranlasst, - so kann es uns nicht wundern, dass unsere anatomischen Kenntnisse von den Nabelbrüchen sich im Grossen und Ganzen noch auf demselben Standpunkte befinden, auf welchen sie durch die schönen Untersuchungen von Sömmerring im Anfange dieses Jahrhunderts gebracht wurden.

Nachdem Söm merring im Jahre 1810 in seiner Preisschrift ${ }^{1}$ ) die wesentlichsten Veränderungen, welche am Nabel nach der Geburt stattfinden, beschrieben hatte, war die anatomische Ursache der Nabelbrüche scheinbar gefunden: die durch fethaltiges Gewebe ausgefüllte Lü̈cke zwischen Nabelvene und oberem Rand des Nabelringes und die Verschiebbarkeit des Peritonäum an dieser Stelle liessen es erklärlich erscheinen, dass unter dazu geeigneten Umständen Ausstälpungen des Bauchfells hier entstehen könnten.

Im Jahre 1856 beschrieb Richet ${ }^{2}$ ) die von ihm entdeckte Fascia umbilicalis und den zwischen dieser und der Linea alba befindlichen Canalis umbilicalis. Es war ganz natürlich, dass Richet dieser Fascie und diesem Kanal eine grosse Bedentung für die Entstehung der Nabelhernien zuschrieb, weil er in diesen anatomischen Verhältnissen Analoga der Fascia infundibuliformis und des Canalis inguinalis zu finden glaubte. Dieser Auffassung entsprach seine Eintheilung der Nabelbrüche in directe und indirecte ${ }^{3}$ ). Nach Richet ist die Fascia umbi-

l) S. Th. Sömmerring, Ueber die Ursache, Erkenntniss und Behandlung der Nabelbrüche. Frankf. a. M. 1811.

2) A. Richet, Du trajet et de l'anneau ombilical, considerés au point de vue de l'anatomie, de la physiologie et de la pathologie. Archives générales de médecine: Decembre 1856 et Janvier 1857.

3) Das Vorkommen der Fascia umbilicalis wurde übrigens schon vor Richet von Vidal de Cassis (Des bernies ombilic. et épigastr. Thèse. 1848) constatirt; auch giebt dieser Autor die Fintheilung der Nabelbrüche in directe und indirecte. Auf p. 60-61 der eben citirten Arbeit heisst es: „J'ai rencontré une bande fibreuse appartenante ar fascia transversalis, qui passe sous la veine orbilicale et près d'ü centimètre au dessus du rebord supérieure de l'anneau. Sous cette bande fibreuse pourraient bien s'engager de petites portions d'épiploon ou d'intestin qui, étraintes, constitueraient de petites hernies interstitielles. On comprend que ces parties puissent .... donner lieu à une bernie, qui serait obhigue de hant en bas. D'autre part il pourrait se produire 
licalis ein das Peritonäum unterpolsterndes fibröses Blatt, welches mit einem scharfen, nach oben concaven Rande oberhalb des Nabelringes beginnt und hart unterhalb desselben endigt; seitlich ist dieses Blatt an die Rectusscheide angeheftet. Bei verschiedenen Individuen sehr ungleich entwickelt, fehlt diese Fascie bei Kindern aus dem ersten Lebensjahre: erst später soll sie unter dem Einflusse der Reibung und des Druckes von Seiten der Eingeweide (?) ihre volle Ausbildung erreichen. Zwischen der Fascie und der linea alba befindet sich ein Kanal (trajet ombilical, gouttière ombilicale), in welchem die in lockeres Fettgewebe eingebettete Vene zum Nabelring verläuft. Durch diesen Kanal wird, nach Richet, bei Erwachsenen der Weg für die Nabelhernie vorgezeichnet, während bei Kindern dieselbe direct durch die Lücke zwischen Vena umbil. und oberem Rande des Nabelringes austritt.

Bald darauf veröffentlichte Robin ${ }^{1}$ ) seine Untersuchungen über die Retraction der Nabelgefässe; unter Anderem berücksichtigte er auch das Verhalten der Fascia umbilicalis. Robin fand dieselbe etwa in der Hälfte der von ihm untersuchten Fälle (bei Kindern aus dem 4. Lebensjahre gut entwickelt); er leugnet jedoch jede Analogie mit den anatomischen Verhältnissen der Leistengegend: in den meisten Fällen sei die Fascie sebr schmal und endige ein paar Centimeter oberhalb des Nabelringes; nur bei kräftigen Individuen finde man die Fascie gut entwickelt, - sie beginne dann etwas unterhalb des Venenstumpfes und erstrecke sich, immer breiter werdend, zuweilen bis zur Höhe der Linea semicirc. Douglas. Eine Bedeutung schreibt $R_{0} b i n$ dieser Fascie nur insofern zu, als sie neben dem nach der Retraction der Gefässe zurückbleibenden ligamentösen Apparat die Resistenz der vorderen Bauchwand in gewissem Grade erböhen könne.

Tillaux ${ }^{2}$ ) bezeichnet das Vorkommen einer Fascia umbilicalis als Seltenheit (Gauderon soll sie in 8 Fällen unter 10

par le même anneau une hernie ordinaire qui serait directe, de sorte que, comme les hernies inguinales, les hernies ombilicales auraient deux varietés : 1) directes, 2) indirectes." (Cit. nach Duplay, De la bernie ombilicale. Thèse. Paris 1866. p. 55.)

1) Robin, Ch., Mémoires de l'acad. impér. de méd. T. 24, 1858.

2) Tillaux, Traité d'anat. topogr. 1884. p. 6ót. 
vermisst haben): die Idee eines Canalis umbilicalis nach Analogie des Canalis inguinalis sei allerdings eine sehr geistreiche, entspreche aber kaum den thatsächlichen Verhältnissen. Das Vorkommen von indirecten Nabelhernien sei nicht gut denkbar; vielmehr gebe die Fettablagerung am oberen Rande des Nabelringes sehr häufig zu Nabelhernien Veranlassung; insofern als der Ring dadurch in seinem oberen Theil erweitert werde.

Féré ${ }^{1}$ ) hat die Fascia umbilicalis schon bei sehr jungen Kindern gesehen, sagt aber nichts über ihre Beziehungen zur Nabelhernie aus; auch führt er die Beobachtungen von Malgaigne, Velpeau, Richard, Marduel u. A. über das Vorkommen der Fascia umbilicalis an.

In Deutschland hat die Entdeckung von Richet mehr Anklang gefunden: die meisten Anatomen und Chirurgen geben zu, dass die Fascia umbilicalis - im Sinne Richet's - mehr oder weniger häufig angetroffen wird, und fassen die Bedeutung dieser Fascie für die Entstehung von Nabelbrüchen ganz ebenso auf, wie das Richet gethan hat. So z. B. sah Kocher ${ }^{2}$ ) in exquisiten Fällen - einmal bei einem 4 Tage alten Knaben - das Peritonäum $2 \mathrm{~cm}$ über dem Nabelring durch den scharfen Fascienrand zu einer queren, nach oben concaven Falte emporgehoben; von hier aus konnte das Peritonäum mit Leichtigkeit zwischen Linea alba und Fascia umbilicalis von oben und hinten nach vorne und unten neben der Vena umbilicalis vorgestülpt werden, welche in dem Canal. umbil. liegt. Kocher zweifelt nicht daran, dass auf diesem Wege Bruchsäcke vorgestülpt werden können, welche durch den Nabelring austreten, und schreibt der Fascia umbilicalis eine analoge Rolle zu, wie der zu einem scharfen concaven Rande verstärkten Fascia transversa am medialen Umfange des hinteren Leistenringes. Ferner hebt Kocher hervor, dass er in zwei Fällen am oberen Rande der Fascia umbilicalis eine deutliche Faltenbildung und eine Grube oberhalb derselben gesehen habe, welche er nach Analogie mit der

1) Féré, Ch., Etudes sur les orifices herniaires et sur les hernies abdominales des nouveau-nés et des enfants à la mamelle. Revue mensuelle de méd. et de chir. 1879 . T. 3.

2) Kocher, Th., Die Hernien in Kindesalter; in Gerhardt's Handbuch der Kinderkrankheiten. Bd. VI. Abth. 2. S. 724 u. 725. Tübingen 1880. 
äusseren Leistengrube als obere Nabelgrube bezeichnen möchte, - im Gegensatz zur unteren Nabelgrube, welche or an die Stelle des Zusammenstossens der drei Nabelgefässe verlegt. Entsprechend diesen beiden Gruben unterscheidet Kocher obere oder indirecte und untere oder directe Nabelbrüche und spricht zum Schluss seiner Betrachtungen über die Fascia umbilicalis die Vermuthung aus, ob nicht vielleicht gerade durch das Fehlen der Fascia umbilicalis eine Hauptdisposition zur Entstehung von Nabelbrüchen gegeben sei.

Wenn ich nun den heutigen Stand der Frage vom Mechanismus der Nabelbruchbildung bei Kindern kurz charakterisiren soll, so heisst es allgemein, dass die Nabelbrüche durch Ausdehnung oder Ausstülpung des Peritonäum von innen her entstehen ${ }^{2}$ ) and durch eine, mit lockerem Fettgewebe ausgefüllte Lücke zwischen Vene, bezw. Venenligament und oberem Umfang des Nabelringes ihren Weg nach aussen finden. Die Bedeutung der Fascia umbilicalis ist weniger klar. Während sie einerseits von Manchen als eine Schutzvorrichtung gegen das Entstehen von (directen) Nabelbrüchen betrachtet wird, lässt man andererseits eine gut entwickelte Fascia umbilicalis als Prädisposition zur Bildung von (indirecten) Nabelhernien gelten.

Auf den Rath meines verehrten Lehrers, Herrn Professor Ed. v. Wahl in Dorpat, habe ich, bei Gelegenheit meiner Untersuchungen über den Processus vaginalis, nach Möglichkeit auch das Verhalten der Fascia umbilicalis studirt. Schon die ersten Sectionen zeigten mir, dass die betreffenden Verhältnisse in mancher Hinsicht von dem, was bisher bekannt geworden war, abweichen, und ich hielt ès daher für zweckmässig, der Sache etwas genauer auf den Grund zu gehen. Da ich nun über ein relativ grosses Untersuchungsmaterial von mehr als 200 Sectionen verfüge, so schien mir die Veröffentlichung der von mir erlangten

1) B. Schmidt (Unterleibsbrüche; in Pitha-Billroth Handbuch. Bd.III. Abth. 2. Lfrg. 3. S. 308) spricht sich folgendermaassen über die Entstehung der Nabelringbrüche aus: "Es ist kaum anzunehmen, dass hierbei lediglich die Bauchpresse den Bruchsack vor sich hertreibt, sondern wahrscheinlicb, dass in jedem Falle das Bauchfell bereits eine gruben-, vielleicht fingerbutförmige Vertiefung darbot, deren Bildung Folge der Veruarbungsvorgänge am Nabel war." 
Resultate wohl berechtigt, um so mehr, als die meisten der in Bezug auf die Fascia umbilicalis angestellten Untersuchungen einen mehr zufälligen Charakter tragen.

Die Fascia umbilicalis kann als ein stärker entwickelter Theil der Fascia transversa aufgefasst werden, welcher die Wi. derstandsfähigkeit des Bauchfells in der Nabelgegend in mehr oder weniger grosser Ausdehnung erhöht. Sie bildet eine aus querverlaufenden Fasern bestehende, mit dem Peritonäum fest verbundene, fibröse Lamelle, welche rechts und links an die Rectusscheide fester angeheftet ist und die Linea alba, sowie alle derselben anliegenden Theile überbrückt. Von der oberen und der unteren Grenze der Fascie sei hier nur soviel gesagt, dass sie in ihrem Verhalten sehr variiren.

Um die Bedeutung der Fascia umbilicalis für die Entwickelung von Nabelbrüchen verstehen zu können, genügt es nicht, dieselbe für sich zu betrachten, sondern es müssen die Beziehungen der Fascie zum Nabelring, zu den Nabelgefässen und zum Peritonäum in Betracht gezogen werden. Diese Beziehungen werden nun in erster Linie durch die Retraction der Gefässe beeinflusst und es ist daher vor Allem nothwendig zu wissen, wie sich die Fascie bei Kindern von verschiedenem Alter, d. h. bei verschiedenen Graden der Retraction der Gefässe verhält.

Wenn man bei einem neugebornen oder nur wenige Tage alten Kinde die peritonäale Fläche der vorderen Bauchwand betrachtet, so erscheint das Peritonäum entsprechend dem Nabelring. und in der nächsten Umgebung desselben ganz glatt und faltenlos. Die Nabelgefässe ziehen unter "dem Peritonäum, dasselbe emporhebend, bis zum Nabelring. Zuweilen ist es bei der Durchsichtigkeit des dünnen Peritonäum möglich, durch dasselbe hindurch die Fasern der Fascia umbilicalis zu sehen. Das Vorkommen der Fascia umbilicalis ist bei Kindern frühesten Alters (von 0-10 Tagen) durchaus keine Seltenheit, - in $\frac{2}{3}$ der untersuchten Fälle war sie deutlich ausgesprochen. Indessen ist sie bei verschiedenen Individuen sehr ungleich entwickelt: zuweilen nur aus wenigen Faserzügen bestehend, bildet sie in einigen Fällen eine breite fibröse Lamelle, welche oberhalb des Nabelringes beginnt und sich noch unterhalb desselben in grösserer 
Ausdehnung verfolgen lässt. Die obere, bezw. die untere Grenze der Fascie ist in vielen Fällen gar nicht mit Sicherheit anzugeben: die Fascie wird allmählich schwächer und geht ohne scharfe Grenze in die Fascia transversa über. In anderen Fällen endigt die Fascie nach unten zu mit einem deutlichen scharfen, nach unten concaven Rand, welcher entweder unterhalb des Nabelringes oder dicht an diesem oder auch oberhalb desselben liegt; nach oben zu verliert sich dabei die Fascie ganz allmählich. Nur ausnahmsweise (3mal unter 23 Fällen) konnte ich an der Fascie einen weniger deutlichen oberen concaven Rand sehen, wie derselbe von Richet und Kocher beschrieben wird; das Peritonäum bildete an dieser Stelle keine Grube.

Die Entfernung des Peritonäum gelingt in den meisten Fällen; nur die Trennung von der Fascia umbilicalis bietet einige Schwierigkeiten, ist jedoch nicht immer so schwer, wie bei älteren Kindern. An der freigelegten Fascie sieht man dasselbe, was bereits bei integrem Peritonäum sichtbar war. Trennt man die Fascie in der Mittellinie, so lässt sie sich sehr leicht von den darunterliegenden Gefässen und der Linea alba nach beiden Seiten hin ablösen bis zu ihrer lineären Insertion auf der Rectusscheide. Die Nabelgefässe erscheinen an der Stelle ihres Zusammenstossens unter einander fest verbunden and verschliessen allseitig den Nabelring, indem sie sich an seiner ganzen Peripherie anheften, so dass man von keiner Seite her durch den Nabelring nach aussen unter die Haut gelangen kann. Allerdings ist die Anheftung der Vene an dem oberen Rande des Ringes in den meisten Fällen nicht so fest, wie die der Arterien: während diese letzteren nicht nur mit der unteren Circumferenz des Ringes, sondern auch mit der Linea alba in der nächsten Nähe des Ringes fest verbunden sind, adhärirt die Vene nicht so fest dem oberen Rande des Nabelringes, und nur ausnahmsweise fehlt diese Adhäsion gänzlich. Jedenfalls ist bei Kindern aus diesem Alter der Nabelring von keiner Seite her zugänglich; selbst in den Fällen, wo die Vene den oberen Rand des Ringes frei lässt, wird die Lücke zwischen diesem und der vorderen Wand der Vene erst dann zugänglich, wenn man die Vene von der Linea alba abhebt.

Nach dem 5.-10. Tag findet man die Arterien bereits etwas 
retrahirt. Ich gehe hier nicht auf die Einzelheiten des Retractionsprozesses ein und verweise den sich dafür Interessirenden auf die sehr eingehende, von Robin gegebene Darstellung der betreffenden Verhältnisse. Es sei hier nur so viel gesagt, dass auch nach der Retraction der Gefässe der Nabelring geschlossen bleibt. Die Arterien retrahiren sich zuerst; die meist verdickte Adventitia derselben bleibt mit der Umrandung des Nabelringes und mit der Vene in Verbindung und bildet einen kleinen, zuweilen mit Eiter oder Blutgerinnseln gefüllten Hohlraum, der nach vorne an den Nabelring grenzt und in vielen Fällen durch eine Oeffnung in der Haut des Nabels nach aussen offen steht. Im unteren Theile dieses Hohlraumes sieht man die beiden Arterienstümpfe, während im oberen Theile die Vene am oberen Rande des Nabelringes nach vorne zur Haut umbiegt. Die hintere Wand des Hohlraumes wird durch die verdickte Adventitia der Arterien gebildet. Sobald sich auch die Vene retrahirt hat, was gewöhnlich erst zwischen dem 20. und 30. Tage deutlich zu sehen ist, ändern sich die oben beschriebenen Verhältnisse nur in so fern, als der von der Venenadventitia gebildete Hohlraum sich auch etwas oberhalb des Nabelringes etablirt und hier den Venenstumpf enthält. Jedenfalls verschliesst aber auch in diesem Stadium die verdickte Adventitia der Nabelgefässe allseitig den Ring. Der von der Adventitia gebildete Hohlraum enthält nicht mehr, wie in den ersten Tagen nach der Geburt, Eiter oder geronnenes Blut, sondern er bildet eine glattwandige Höhle, welche nach aussen durch die Nabelnarbe geschlossen wird.

Das Verhalten der Fascia umbilicalis zeigt im Laufe des ersten Lebensmonats keine Veränderungen, - höchstens dass sie bei älteren Individuen deutlicher differenzirt ist, als in den ersten Tagen nach der Geburt; im Uebrigen bietet sie dieselben, oben bereits beschriebenen, individuellen Verschiedenheiten. Das Peritonäum erscheint fester mit der Fascie verbunden, bildet aber keinerlei Falten oder Gruben. Folgende Zusammenstellung giebt über das Vorkommen, bezw. Verhalten der Fascie bei Kindern aus dem ersten Lebensmonat Aufschluss:

Unter 92 Fällen fehlte die Fascia umbilicalis $34 \mathrm{mal}$; in 58 Fällen war sie mehr oder weniger deutlich ausgesprochen. Dabei war die Fascie: 


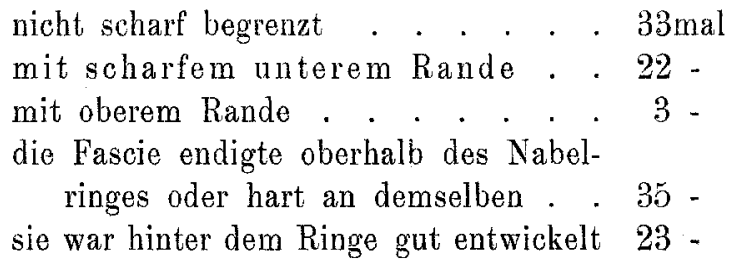

Nach vollendetem erstem Lebensmonat beginnt die Umwandlung der nach der Retraction zurückbleibenden 'Tunica adventitia der Nabelgefässe in eine Anzahl dünner Stränge, deren Anordnung und Verbindung unter einander, sowie mit der Umgebung des Nabelringes von Robin sehr eingehend beschrieben worden ist. Schon zu Ende des ersten Monats wird der oben beschriebene, von der Adventitia gebildete Hohlraum immer seltener angetroffen; die Adventitia selbst ist bereits dünner geworden und zieht als ein breiter Strang über den Nabelring hinweg von dem Venenendstück zu den Arterienstümpfen; zu Anfang des zweiten Monats steht dieser von der Adventitia gebildete Strang in den meisten Fällen noch mit der Peripherie des Ringes in Verbindung und verschliesst denselben, wenn auch nicht so sicher, wie das bei jüngeren Kindern der Fall war. Mit fortschreitendem Alter zerfällt der oben erwähnte einzelne Strang in mehrere dünnere, welche den Nabelring entweder ganz frei lassen oder nur zum Theil verschliessen.

Die Fascia umbilicalis zeigt dieselben Eigenthümlichkeiten, wie bisher, nur ist der untere Rand in den Fällen, wo er vorhanden ist, viel schärfer ausgesprochen, als bei jüngeren Individuen, und hebt in den meisten Fällen das Peritonäum zu einer leichten, nach unten concaven Falte empor. Ausserdem liegt wenigstens ein Theil der Fascie nach der Retraction der Gefässe der Linea alba inniger an, von welcher sie früher durch die Nabelgefässe abgehoben war.

Die wesentlichsten Veränderungen, welche im Verhalten des Nabelringes und der Fascie durch die Retraction der Gefässe hervorgebracht wurden, lassen sich in wenigen Worten zusammenfassen: der Nabelring hat den durch die Gefässe, bezw. deren verdickte Adventitia gegebenen Schutz verloren; er liegt nun mehr oder weniger frei und ist nur in denjenigen Fällen ge- 
schützt, in welchen die Fascia umbilicalis unterhalb des Ringes endigt. Ein Theil der Fascie liegt in der Nabelgegend der Linea alba dicht an und deckt die nach der Retraction zurückbleibenden dünnen Gefässstränge.

Wir sehen somit, dass die Bedeutung der Fascie als Schutzvorrichtung erst dann zur vollen Geltung kommt, wenn die Gefässe sich mehr oder weniger retrahirt haben und mit dem Nabelring nur durch ein Paar dünne Stränge, welche denselben meist nicht verschliessen, in Verbindung stehen. Es ist daher hier am Platze, die verschiedenen Formen, welche die Fascia umbilicalis bei Kindern nach vollendetem erstem Lebensmonat darstellt, genauer zu beschreiben; ich theile diese Formen in regelmässige und unregelmässige ein und beginne mit den ersteren.

Regelmässige Formen der Fascia umbilicalis:

1) Die Fascia umbilicalis bildet eine breite fibröse Lamelle, welche ohne scharfe Grenze oberhalb des Nabelringes beginnt und ebenso allmählich unterhalb desselben verschwindet. Dem Nabelring entsprechend ist die Fascie am stärksten entwickelt. Das Peritonäum ist fest mit der Fascie verbunden und auf derselben weder faltbar noch verschiebbar: es streicht glatt über den Nabelring hinweg, ohne die geringste Einsenkung, geschweige denn eine Ausstülpung zu zeigen. Die Entstehung einer Nabelhernie scheint mir in solchen Fällen unmöglich, - einfach wegen der Unverschiebbarkeit des Peritonäum; ausserdem dürfte auch die straffe Fascie dem Nabelring einen hinreichenden Schutz gewähren.

2) Am oberen Ende der Fascie findet man (sehr selten) die Andeutung eines nach oben concaven Randes, welcher die Vene sichelförmig umgreift (Taf. IV Fig. 1). In zwei Fällen sah ich an dieser Stelle eine flache Peritonäaltasche, die aber sofort verschwand, wenn man die Vene etwas anzog. Ich glaube, dass diese Tasche sich erst bildete, nachdem die Vena umbilicalis nahe der Porta hepatis durchtrennt worden war: die Vene zog sich dabei etwas gegen den Nabelring zurück und natürlicherweise musste sich das mit der Vene verbundene Peritonäum an einem festeren Theile der Fascie falten. Nach dieser Auffassung wäre also die oben erwähnte Peritonäaltasche am oberen Rande der Fascie als eine in Folge der Präparation entstandene zu betrachten. 
3) Bei weitem häufiger ist die untere Grenze der Fascie durch einen scharfen, nach unten concaven, leicht vorspringenden Rand gekennzeichnet. In den Fällen, wo dieser Rand unterhalb des Nabelringes liegt, bleiben die Verhältnisse genau dieselben, wie bei den sub 1 und 2 beschriebenen Formen, d. h. der Ring ist vollkommen geschützt. Die Verhältnisse ändern sich aber, sobald der Fascienrand dem Nabelring näher zu liegen kommt. Wir haben dann in der nächsten Nähe des Nabelringes zwei neben einander liegende, durch deu scharfen Fascienrand von einander abgegrenzte Peritonäalabschnitte: der obere (bis zum Nabelring) ist mit der Fascie fest verbunden und weder verschiebbar noch dehnbar, der untere (unterhalb des Ringes) liegt der Linea alba, bezw. den Arteriensträngen auf und ist sehr leicht zu verschieben. Eine sehr unzweideutige Folge dieser Verschiebbarkeit habe ich in mehreren Fällen gesehen: in einem Falle (Taf. V Fig. 1) lag der sehr scharfe Rand der breiten Fascie hart unterhalb des Ringes; der verschiebbare Theil des Peritonäum hatte sich am Rande der Fascie nach oben zwischen Fascie und Linea alba eingestülpt und bildete ausserdem ein sackförmiges Divertikel durch den Nabelring. Ich konnte nicht mit Sicherheit eruiren, ob eine Hernie bei Lebzeiten bemerkt worden war. - In zwei anderen Fällen lag der Fascienrand hart am Nabelring, so dass die Fascie den Ring in seiner oberen Hälfte verdeckte: in beiden Fällen (Taf. V Fig. 3 u. 4) war ein Divertikel durch den Ring vorhanden, sowie eine Ausstülpung nach oben zwischen Fascie und Linea alba; in einem dieser Fälle (Fig. 4) war sicher eine Hernie constatirt, die mit einem einfachen Heftpflasterverbande behandelt worden war.

4) Sehr häufig endigt die Fascie mit einem scharfen concaven Rande oberhalb des Nabelringes in einer Entfernung von $0,5-2,5 \mathrm{~cm}$ von demselben. Bei dieser Form der Fascie bleibt der Nabelring ohne Schutz, abgesehen von denjenigen Fälleu, wo er durch die Gefässstränge in einer, allerdings mangelhaften Weise geschlossen wird. Das Peritonäum ist unterhalb des Fascienrandes, entsprechend dem Nabelring und in der nächsten Umgebung desselben, leicht verschieblich und bildet häufig ein Divertikel durch den Ring. Dagegen ist das Peritonäum mit der Fascie sehr fest verbunden und kann auf derselben absolut 
nicht versehoben werden. An der Grenze des versehiebbaren und nicht verschiebbaren Theils, also am Fascienrande, bildet das Peritonäum zuweilen eine nach oben geschlossene, bis $0,5 \mathrm{~cm}$ tiefe Tasche, welche zwischen Fascie und Linea alba hinaufgeht und durch keinerlei Manipulationen ausgeglichen werden kann. Sehr oft combinirt sich das Vorkommen einer solchen Peritonäaltasche mit einer Divertikelbildung am Nabelring.

Die hier erwähnten Divertikel sind einer genaueren Betrachtung wohl werth, denn sie stehen jedenfalls zu der Nabelbruchbildung in naher Beziehung; sie können unter allen Umständen entweder als in der Bildung begriffene oder dem Verschwinden entgegensehende Bruchsäcke aufgefasst werden, obgleich es schwer sein dürfte, in jedem Falle zu entscheiden, um welche dieser beiden Möglichkeiten es sich handelt. Das Vorkommen von Divertikeln durch den Nabelring wurde bereits von Malgaigne und Klebs constatirt und als ein wesentliches ätiologisches Moment für die Entstehung von Umbilicalhernien angesehen. Malgaigne ${ }^{1}$ ) wollte eine besondere Abart von angeborenen Nabelbrüchen bei Kindern mit stark prominirendem Hautnabel unterscheiden, bei welchen das Peritonäum "par excès de développement" ein angeborenes Divertikel durch den Nabelring bildete. Klebs ${ }^{2}$ ) hat zuweilen bei Neugeborenen leere Ausstülpungen des Peritonäum gefunden: „es dürfte also auch hier wie bei den angeborenen Leistenbrüchen die Peritonäalausstülpung aus dem Fötalleben mit hinübergenommen und nur das Hineintreten der Eingeweide später erfolgt sein. Es stimmt dies überein mit der bekannten Erfahrung, dass diese Brüche plötzlich beim Schreien entstehen".

Was die von mir beobachteten Divertikel anbetrifft, so sehe ich keine Veranlassung, dieselben als angeborne zu betrachten: der Umstand, dass das jüngste Kind, bei welchem sich ein Divertikel vorfand, über 6 Wochen alt war, sowie die Coincidenz der Divertikel mit einer ganz bestimmten Fascienform macht vielmehr die Annahme wahrscheinlich, dass wir es hier mit erworbenen Divertikeln zu thun haben. Das Vorkommen von angeborenen Divertikeln muss jedenfalls eine Seltenheit sein und

1) Leçons cliniques sur les hernies. p. 222. Paris 1841.

2) Handbuch der pathologischen Anatomie. Lfrg. 2. S. 214. Berlin 1869 
kann für die Aetiologie der Nabelbrüche nicht wesentlich in Betracht kommen.

Die erworbenen Divertikel im Nabelring bieten in ihrem Verhalten mancherlei individuelle Verschiedenheiten. Die Eingangsöffinung entspricht ihrer Grösse und Form noch ziemlich genau dem Nabelring und zeigt eine mehr oder weniger scharfe Umrandung: zuweilen findet man, dass an einem Theile der Peripherie die Oeffnung schärfer begrenzt ist. Das Divertikel selbst ist verschieden gross und hat eine eylindrische, ovoide oder kugelförmige Gestalt. Mit der Nabelnarbe ist die Wand des Divertikels in den meisten Fällen so verbunden, dass die Trennung ohne besondere Schwierigkeiten gelingt. Die Venenstränge haben zum Divertikel keine Beziehung, indem sie sich zu beiden Seiten des Nabelringes anheften und den Ring ganz frei lassen. In denjenigen Fällen, wo noch ein dritter mittlerer Strang zur oberen Peripherie des Ringes hinzieht, verliert er sich meist in der oberen Wand des Divertikels; übrigens ist dieser Strang so dünn, dass er sich nur in wenigen Fällen deutlich verfolgen lässt. Die Arterienstränge heften sich an die untere Peripherie des Nabelringes an und verlaufen in manchen Fällen an der unteren Wand des Divertikels zur Nabelnarbe. -

\section{Unter den}

unregelmässigen Formen der Fascia umbilicalis fasse ich eine geringe Anzahl von Fällen - meist bei Kindern nach dem 3. Monat - zusammen, in welchen die Anordnung der Fascie insofern eine abweichende ist, als sie hie und da Lücken aufweist oder aus mehreren, von einander getrennten Abschnitten besteht. So z. B. fand ich in 3 Fällen eine sonst regelmässige Fascie, die sich bis unterhalb des Ringes erstreckte und genau entsprechend dem Nabelring eine ovale Lücke zeigte, durch welche in 2 Fällen das Peritonäum in Form eines kleinen Divertikels ausgestülpt war. In 2 anderen Fällen fehlte die Fascie, dem Ring entsprechend, gänzlich; sie endigte oberhalb des Ringes mit einem scharfen, nach unten concaven Rande, welcher seitlich vom Nabelring in den nach oben coneaven Rand des unteren Abschnittes der Fascie überging. In dem in Fig. 4 (Taf. IV.) abgebildeten Falle bildete das Peritonäum ein kleines Divertikel durch den Ring und 2 
durch eine vertical stehende Peritonäalfalte getrennte Taschen am Rande des oberen Abschnitts der Fascie. In einem anderen Falle (Taf. IV, Fig. 2) bildete die oberhalb des Nabelringes regelmässig entwickelte Fascie, dem Ring entsprechend und unterhalb desselben, ein zierliches Netzwerk mit horizontal gelagerten spindelförmigen Maschen; durch eine dieser Maschen stülpte sich das Peritonäum divertikelartig nach aussen.

Eine besondere Gruppe bilden die Fälle, in welchen eine besonders differenzirte Fascia umbilicalis fehlt, $d$. h. wo die Fascia transversa an der vorderen Bauchwand überall gleichmässig entwickelt ist. In diesen Fällen ist das Peritonäum in der Gegend des Nabelringes, sowie oberhalb und unterhalb desselben, gleichmässig verschiebbar, wenn auch meist nur in geringem Grade; dabei ist das Peritonäum ausnahmslos glatt und bietet weder Falten noch Ausstülpungen an der vorderen Bauchwand. Nur in einem Falle bildete es, dem Ring entsprechend, eine flache Einsenkung. Der Nabeiring ist entweder ganz frei oder zum Theil durch die Gefässstränge verdeckt.

Folgende Zahlen geben eine annähernde Vorstellung von dem Vorkommen der oben beschriebenen Formen der Fascia umbilicalis bei Kindern aus dem 2. bis 11. Lebensmonat.

Unter 115 Fällen fehlte die Fascie gänzlich 30 mal; das Peritonäum dabei ganz glatt; nur in einem Fall war eine flache Einsenkung am Ring.

Die Fascie deutlich sichtbar in 85 Fällen:

Sie deckte den Nabelring in 25 Fällen.

nicht scharf begrenzt $7 \mathrm{mal}$,

scharfer unterer Rand 9mal,

oberer Rand angedeutet $5 \mathrm{mal}$,

unregelmässig entwickelt $5 \mathrm{mal}$; dahei $2 \mathrm{mal}$ Peritonäaldivertikel durch eine Lücke in der Fascie.

(nicht scharf begrenzt $7 \mathrm{mal}$; dabei 2mal Divertikel durch den Ring.

Der untere Rand scharf 4:9mal, und liegt:

Der Nabelring war von der Fascie nicht gedeckt in 60 Fällen.
1) $0,5-2,5 \mathrm{~cm}$ über dem Ring 30mal: daruuter $3 \mathrm{mal}$ oine Peritonäalfalte am Rande der Fascie, 12 mal Peritonäaldivertikel mit oder ohne Peritonäalfalte und $4 \mathrm{mal}$ eine Hernie.

2) hart am oberen Rande des Nabelringes oder denselben zum Theil überragend $19 \mathrm{mal}$ : darunter 1 mal Peritonäalfalte, 6mal Divertikel, meist mit Peritonäalfalte, und smal eine Hernie.

Der obere Rand angedeutet in 4 Fällen.

Unregelmässige Anordnung $4 \mathrm{mal}$; dabei $2 \mathrm{mal}$ Divertikel und lmal Peritonäalfalte. 
Eine kurze Betrachtung der hier gegebenen Zusammenstellung lässt uns das Vorkommen der verschiedenen Formen der Fascia umbilicalis, zugleich aber auch deren Bedeutung für das Zustandekommen von Umbilicalhernien bei Kindern beurtheilen. Ich habe bereits oben darauf hingewiesen, dass bei Kindern aus dem ersten Lebensmonat der Nabelring durch die Gefässtümpfe fest verschlossen ist und dass die Fascia umbilicalis in diesem Alter lange nicht die Bedeutung haben kann, wie im späteren Alter: die Entstehung einer Umbilicalhernie ist durch den Gefässverschluss nahezu unmöglich gemacht, ganz gleich ob eine Fascia umbilicalis da ist oder nicht. Nach erfolgter Retraction der Gefässe ändert sich die Sachlage wesentlich, indem der Nabelring frei und zur Bildung von Peritonäaldivertikeln geeignet wird. Bei solchen Individuen (nach vollendetem erstem Lebensmonat) kann diese oder jene Anordnung der Fascie auf die Möglichkeit einer Umbilicalhernie von Einfluss sein.

Als wesentliche Momente, welche der Fascia umbilicalis eine Bedeutung für die Entstehung von Nabelhernien vindiciren, betrachte ich die eigene Festigkeit der Fascie und ihren Einfluss auf die Verschiebbarkeit des Peritonäum. Was den ersteren Punkt anlangt, so habe ich mich in einer Reihe von Fällen überzeugt, dass die Fascia umbilicalis beim Versuche, sie zu dehnen, einen ziemlich erheblichen Widerstand leistet. Wenn man die Linea alba in der Längsrichtung spaltet und das Peritonäum, bezw. die Fascia umbilicalis von vorne her freilegt, so fühlt man beim Herüberstreichen mit dem Finger einen deutlichen Widerstand an denjenigen Stellen, wo die Fascia umbilicalis wenn auch nur mässig entwickelt ist. Was ferner die Verschiebbarkeit des Peritonäum an der vorderen Bauchwand anbetrifft, so habe ich bereits oben gezeigt, dass dasselbe mit der Fascie fest verwachsen ist und in Folge dessen an denjenigen Stellen, wo die Fascie vorhanden, absolut nicht verschiebbar ist.

Daraus geht hervor, dass bei Individuen, bei welchen die Fascia umbilicalis den Nabelring verdeckt, die Disposition zu Nabelhernien eine sehr geringe sein muss. In der That fand sich bei dieser Anordnung der Fascie keine einzige Hernie; nur $2 \mathrm{mal}$ war ein kleines Peritonäaldivertikel vorhanden, und in beiden Fällen zeigte die Fascie entsprechend dem Nabelring 
eine Lücke. Die Festigkeit der Fascie und die Unverschiebbarkeit des Peritonäum macht die Entstehung einer Hernie bei solchen Individuen nahezu unmöglich.

Unter den anderen Formen der Fascia umbilicalis zeichnet sich eine ganz besonders aus durch ihre häufige Coincidenz mit Peritonäalfalten und Divertikeln, - es sind das die Fälle, in welchen die Fascie entweder hart am Nabelring oder oberhalb desselben mit einem scharf nach unten concaven Rande endigt. Es liegt sehr nahe, das häufige Vorkommen von Peritonäalfalten, bezw. Divertikeln in causale Beziehung zu dieser Anordnung der Fascie zu setzen und die letatere als ein wesentliches prädisponirendes Moment für die Entstehung von Nabelbrïchen anzusehen. Schon die Thatsache, dass sämmtliche Fälle, wo ein Nabelbruch sicher constatirt werden konnte, grade diese Anordnung der Fascie zeigten, berechtigt uns zu der soeben aufgestellten Behauptung. Es wäre nur noch wünschenswerth, den Mechanismus des Zustandekommens von Divertikeln und Peritonäalfalten bei der in Rede stehenden Form der Fascie nach Möglichkeit aufzuklären.

Wenn die oben genauer beschriebenen Peritonäaldivertikel mit grosser Wahrscheinlichkeit als erworbene aufgefasst werden kounten, so gilt dasselbe wohl ganz unzweifelhaft von den Peritonäalfalten, bezw. Peritonäaltaschen, welche häufig am Rande der Fascie angetroffen werden. Die Entstehung dieser Peritonäaltaschen könnte auf ungleichmässige Verschiebungen des Peritonäum an der vorderen Bauchwand zurückgeführt werden. Bei den Respirationsbewegungen, sowie bei jeder Anstrengung der Bauchpresse wird die Fläche der vorderen Bauchwand alternirend verkleinert und vergrössert. Das Peritonäum wird dabei vermöge seiner Elasticität diesen Bewegungen nachfolgen, ohne sich irgend wo zu falten, wenn es nur überall gleichmässig verschiebbar und dehnbar ist. In der That sehen wir auch keine Faltenbildunğ an der vorderen Bauchwand, sobald eine besondere Fascia umbilicalis vorhanden ist: das Peritonäum verhält sich in diesen Fällen ähnlich, wie der seröse Ueberzug der Darmschlingen, welcher, ohne sich zu falten, ziemlich bedeutende Schwankungen in seiner Flächenausdehnung vertragen kann. Anders steht die Sache in den Fällen, wo ein begrenzter Theil des Bauchfells 
durch das Vorhandensein einer Fascie unverschiebbar geworden ist, - es liegt auf der Hand, dass der verschiebbare Theil des Peritonäum sich aun unter Umständen am scharfen Rande der Fascie falten und zur Taschenbildung Veranlassung geben kann.

Andererseits ist es aber auch möglich, dass bei der Retraction der Nabelvene ein Theil des ihr anhaftenden Peritonäums unter die Fascie mitgezogen wird ${ }^{1}$ ). Welche dieser beiden Möglichkeiten bei der Bildung von Peritonäaltaschen besonders in Frage kommt, will ich unentschieden lassen, - soviel steht jedoch fest, dass die Entwickelung der Fascie oberhalb des Nabelringes und das Vorhandensein eines scharfen, nach unten concaven Fascienrandes die Falten-, bezw. Taschenbildung am Peritonäum ganz besonders begünstigt.

Dieselbe Anordnung der Fascie erleichtert auch die Bildung von Peritonäaldivertikeln durch den Nabelring and zwar, wie mir scheint, auf Grund folgender mechanischer Bedingungen:

1) Die durch die Fascie erhöhte Widerstandsfähigkeit, bezw. verminderte Dehubarkeit der vorderen Bauchwand muss nothwendig eine Steigerung des continuirlichen intraabdominalen Druckes zur Folge haben. Bei gegebenem Füllungszustande des Magendarmkanals wird der intraabdominale Druck um so höher ausfallen, je grösser die Widerstände sind, welche die Bauchwand der Ausdehnung des Magendarmkanals bietet.

2) Da ein Theil des Peritonäum durch die Fascie undehnbar und unverschiebbar gemacht worden ist, so wird bei einem gegebenen Druck der nachgiebige Theil des Peritonäum mehr gedehnt und verschoben werden, als in den Fällen, wo das Peritonäum an der ganzen vorderen Bauchwand gleichmässig dehnbar und verschiebbar ist. Die Wirkung des intraabdominalen Druckes concentrirt sich gewissermaassen auf den Nabelring und dessen nächste Umgebung und das Peritonäum ist hier einer stärkeren Dehnung und Verschiebung ausgesetzt.

Es bedarf keiner weiteren Auseinandersetzung, dass unter solchen Umständen Ausstülpungen des Bauchfells durch den Nabelring leichter zu Stande kommen werden, als bei fehlender

1) Für diese Möglichkeit würde der Umstand sprechen, dass die Faltenbildung nicht beobachtet wird, wenn die Gefässe sich noch nicht retrahirt haben oder wenn der Fascienrand unterhalb des Nabelringes liegt. 
Fascie, wo ein relativ geringerer Druck in seiner Wirkung auf eine grössere Fläche vertheilt ist. Diese Ausstülpungen können nun als die ersten Anfänge einer Nabelhernie betrachtet werden. Wenn nicht rechtzeitig eingeschritten wird, so werden diese Ausstülpungen grösser und bilden schliesslich zur Aufnahme von Eingeweiden geeignete Bruchsäcke. Das zur Bildung des Bruchsackes nöthige Material wird zunächst von der nächsten Umgebung des Nabelringes hergenommen, während später höchst wahrscheinlich das sehr verschiebbare, unterhalb des Nabelringes gelegene Peritonäum mit hineingezogen wird. Das oberhalb des Nabelringes liegende Peritonäum kann zur Bruchbildung nicht benutzt werden, weil es durch die Fascie unverschiebbar gemacht ist. Die Venenstränge zeigen ein verschiedenes Verhalten, indem sie zum Theil sich zu beiden Seiten des gedehnten Nabelringes anheften, zum Theil eine Strecke weit an der äusseren Fläche des Bruchsackes verlaufen, was bei den Arteriensträngen constant der Fall ist.

In den Fällen, wo die Fascie oberhalb des Nabelringes endigt, bildet dieser die Bruchpforte: die Brüche treten direct durch den Ring nach aussen (Taf. V Fig. 2), - nicht durch eine Lücke zwischen Vene und oberem Umfang des Nabelringes. Zu der Zeit, wo die grösste Zahl von Nabelhernien zu entstehen pflegt, d. h. zu Anfang des zweiten Monats, hat die Nabelvene nichts mit dem Nabelring zu thun und die dünnen Stränge, welche rom Venenstumpf zum Ring hinziehen, sind eben viel zu dünn, als dass sie auf die Austrittsstelle des Bruches von Einfluss sein könnten.

Wenn der untere Fascienrand hart am Nabelring liegt oder gar den oberen Theil desselben verdeckt, so wird die Bruchpforte einerseits von dem unteren Umfang des Nabelringes und andererseits von dem scharfen Rande der Fascie gebildet; in solchen Fällen besitzt der Bruchsack zuweilen ein Divertikel, welches nach oben zwischen Fascie und Linea alba hinaufgeht. Obgleich Nabelbrüche bei Kindern selten eingeklemmt werden, so wird doch durch das zuletzt geschilderte anatomische Verhalten der Gedanke an die Möglichkeit einer Einklemmung zwischen unterem Umfang des Nabelringes und Fascienrand nahe gelegt. Desgleichen könnte eventuell der Fall eintreten, dass Archiv f, pathol. Anat. Bu. CVII. Hft. 1. 
man bei der Reposition eines Nabelbruches seinen Inhalt statt in die Bauchhöhle in ein Divertikel zwischen Fascie und Linea alba hineinschiebt.

Folgende Sätze enthalten, kurz gefasst, die wesentlichen Re. sultate meiner Untersuchung:

1) Die Fascia umbilicalis ist ein stärker entwickelter Theil der Fascia transversa, welcher bei Kindern von der Geburt an bis gegen das Ende des ersten Lebensjahres zwar sebr ungleich entwickelt, aber doch sehr häufig angetroffen wird; sie ist im Allgemeinen bei älteren Kindern besser differenzirt, als in der ersten Zeit nach der Geburt.

2) Sie bildet in exquisiten Fällen ein fibröses Blatt, welches rechts und links auf der Rectusscheide in einer fast vertical herablaufenden Linie angeheftet ist. Die obere Grenze ist nur ausnahmsweise durch einen sichtbaren Rand angedeutet; dagegen besitzt die Fascie sehr häufig einen unteren scharfen, nach unten concaven Rand, an welchem das Peritonäum zuweilen zu einer Falte emporgehoben wird oder gar eine zwischen Fascie und Linea albaliegende Tasche bildet.

3) Je nach der Lage und Beschaffenheit der Fasciengrenzen lassen sich verschiedene Formen der Fascia umbilicalis unterscheiden, deren Bedeutung für die Entstehung von Nabelhernien wesentlich davon abhängt, ob der Nabelring von der Fascie gedeckt ist oder nicht. Die ätiologische Rolle der Fascia umbilicalis wird hauptsächlich bedingt durch ihre Widerstandsfähigkeit, sowie durch den Umstand, dass der ihr fest anhaftende Theil des Peritonäum gänzlich unverschiebbar und undehnbar ist.

4) Bei Kindern aus dem ersten Lebensmonat hat die Fascia umbilicalis keine wesentliche Bedeutung, weil der Nabelring durch die Gefässe, bezw. deren verdickte Adventitia so vollkommen geschlosen wird, dass die Bildung eines Nabelbruches nur ausnahmsweise möglich sein dürfte.

5) Nach vollendetem erstem Lebensmonat ist der Nabelring mehr oder weniger frei. Als Prädisposition zur Nabelhernie ist dann diejenige Form der Fascie zu betrachten, bei welcher der untere scharfe Rand entweder 
hart am Nabelring oder etwas oberhalb desselben liegt. Bei dieser Form der Fascie werden am bäufigsten Falten, bezw. Taschen am Peritonäum und Divertikel durch den Ring, sowie vollkommen ausgebildete Hernieu angetroffen. Viel weniger prädisponirt sind diejenigen Individuen, bei welchen die Fascia umbilicalis gänzlich fehlt, während in denjenigen Fällen, wo der Ring durch die Fascie geschlossen wird, die Entstehung einer Nabelhernie nahezu unmöglich erscheint.

6) Nabelbrüche treten immer direct durch den Nabelring aus; zur Bildung des Bruchsackes wird mehr das unterhalb des Nabelringes gelegene, verschiebbare Peritonäum benutzt. Wenn der Fascienrand hart am Nabelring liegt oder denselben etwas verdeckt, so bildet er die obere Umrandung der Bruchpforte; in solchen Fällen besteht zuweilen ein Divertikel, welches nach oben zwischen Fascia und Linea alba hinaufgeht.

\section{Erklärung der Abbildungen.}

Tafel IV und V.

In den vorliegenden Abbildungen ist die peritonäale Fläche eines Theils der vorderen Bauchwand bei integrem Peritonäum dargestellt. Die frischen oder in Weingeist aufbewahrten Objecte wurden vor dem Zeicbnen keiner Präparation unterworfen; nur in. Fig. 1 und 2 der Taf. $V$ war die vordere Bauchwand in der Linea alba fast bis zum Nabelring getrennt und die Fascie mit der ihr anliegenden Vene von vorne her freigelegt.

$$
\text { Tafel IV. }
$$

Fig. 1. Alter des Kindes 3 Monate 9 Tage. Die Fascie verschliesst den Nabelring. Der obere Rand ist schärfer als der untere.

Fig. 2. Alter 3 Monate 12 Tage. Unregelmässige Fascie. Divertikel im Nabelring.

Fig. 3. Alter 4 Monate. Der scharfe untere Rand der Fascie ca. $1 \mathrm{~cm}$ über dem Nabelring. Divertikel.

Fig. 4. Alter 3 Monate 23 Tage. Unregelmässige Fascie, welche den Ring frei lässt. Am unteren Rande des oberen Abschnitts der Fascie bildet das Peritonäum zwei Taschen, welche durch eine vertical stehende Peritonäalfalte (P f) von einander getrennt sind. Divertikel im Ring. Tafel $V$.

Fig. 1. Alter 2 Monate 4 Tage. Breite Fascie. Der obere Rand kaum angedeutet. (Der scharf gezogene bogenförmige Rand im obersten Theil der Zeichnung gehört nicht der Fascie, sondern einem bei der Präparation entstandenen Riss des Peritonäum.) Der untere Rand sebr scharf, liegt am unteren Umfang des Nabelringes. Das Perito- 
nåum bildete hier eine nach oben führende Tasche und ein Divertikel im Ring.

Fig. 2. Alter 5 Monate. Hernia umbilicalis. Der Fascienrand scharf, etwa $2,5 \mathrm{~cm}$ über dem weiten Nabelring.

Fig. 3. Alter 3 Monate 5 Tage. Scharfer unterer Fascienrand hart am Nabelring. Peritonäaltasche und Divertikel.

Fig. 4. Alter 1 Monat 20 Tage. Die Fascie verdecikt den oberen Theil des Nabelringes. Scharfer Rand; Peritonäaltasche nnd Divertikel im Ring. Bei Lebzeiten wurde eine Hernia umbilicalis constatirt.

Die Bezeichnungen sind auf beiden Tafeln dieselben:

F Fascia umbilicalis. Fr Rand der Fascie. Nr Nabelring. Vu Vena umbilicalis. Vl Venenligament. Al Arterienligament.

\section{VII. \\ Kleinere Mittheilungen.}

1.

\section{Ein weiterer Fall von circumscripter Miliartuberculose in der offenen Lungenarterie.}

Von Dr. Karl Herxheimer,

früherem Assistenten am Senckenbergischen pathologischen Institut zu Frankfurt a. M.

Dem unlängst von Herrn Prof. Weigert mitgetheilten Fall von Tuberculose der Lungenarterienintima (dieses Arch. Bd. 104. 1886. S. 31) folgt ein zweiter aus dem Sectionsmateriale des Senckenbergischen Instituts, bei welchem ebenfalls in einer grossen offenen Lungenarterie eine umschriebene für das blosse Auge eben noch erkennbare Miliartuberculose sich vorfand. Bei der grossen Seltenheit derselben ist es gewiss gerechtfertigt, die Literatur um einen Fall zu erweitern.

Fräulein K., 23 Jahre alt.

Sebr abgemagerter Leichnam. Ganz leichtes Oedem der Knöchel.

In der rechten Pleurahöhle eine grössere Menge gelbröthlichen Exsudates mit Fibrinflocken gemischt.

Das Herz von entsprechender Grösse, Musculatur blass, Klappen zart.

In der Spitze der linken Lunge einige erbsengrosse käsige Heerde. Weiter nach unten zerstreute Gruppen käsiger Knötchen, im Ganzen sehr wenig. Auch im Unterlappen ganz vereinzelte miliare käsige Heerde. In einigen kleineren Arterien graurothe, der Wandung fest anhaftende Gerinnsel.

Auf der rechten Lunge ist die Pleura mit reichlichen Fibrinmassen belegt. Im Oberlappen zahlreiche käsige zum Theil zerfallene Heerde, einige 